\title{
PilP, a pilus biogenesis lipoprotein in Neisseria gonorrhoeae, affects expression of PilQ as a high-molecular-mass multimer
}

\author{
Sandra L. Drake, ${ }^{\dagger}$ Sara A. Sandstedt ${ }^{\ddagger}$ and Michael \\ Koomey* \\ Department of Microbiology and Immunology, University \\ of Michigan Medical School, Box 0620, Ann Arbor, \\ Michigan 48109, USA.
}

\section{Summary}

Studies of gonococcal pilus biogenesis are fundamental to understanding organelle structure/function relationships and identifying new approaches to controlling disease. This area of research is also relevant to elucidating the basic mechanisms of outer membrane translocation of macromolecules, which requires components highly related to those involved in type IV pilus expression. Previous studies have shown that products of several ancillary pil genes are required for organelle biogenesis but of these only PilQ, a member of the GspD protein family, is a component of the outer membrane. DNA sequencing of the region upstream of pilQ revealed the presence of two open reading frames (ORFs) whose deduced polypeptides shared significant identities with proteins required for pilus expression in Pseudomonas aeruginosa and Pseudomonas syringae, the genes for which are arrayed upstream of a gene encoding a PilQ homologue. Gonococcal mutants bearing transposon insertions in these ORFs were non-piliated and failed to express pilus-associated phenotypes, and the corresponding genes were designated pilO and pilP. The piliation defects in the mutants could not be ascribed to polarity on distal pilQ expression as shown by direct measurement of PilQ antigen in those backgrounds and the use of a novel technique to create tandem duplications in the gonococcus (Gc) genome. As predicted by the presence of a consensus lipoprotein signal sequence, PilP expressed in both Escherichia coli and Gc could be labelled with $\left[{ }^{3} \mathrm{H}\right]$-palmitic acid. PilP ${ }^{-}$as well as PilQ ${ }^{-}$mutants shed PilC, a protein which facilitates pilus assembly and is implicated

Received 6 August, 1996; revised 18 November, 1996; accepted 21 November, 1996. Present addresses: †Eastern Michigan University, Ypsilanti, Michigan, USA; ‡Department of Biology, University of Michigan, Ann Arbor, Michigan, USA. *For correspondence. E-mail mkoomey@umich.edu; Tel. (313) 7633672; Fax (313) 7643562. in epithelial cell adherence, in a soluble form. Combined with the finding that levels of multimerized PilQ were greatly reduced in PilP $^{-}$mutants, the results suggest that PilP is required for PilQ function and that PilQ and PilC may interact during the terminal stages of pilus biogenesis. The findings also support the hypothesis that the Gc PilQ multimer corresponds to a physiologically relevant form of the protein required for pilus biogenesis.

\section{Introduction}

Expression of type IV pili or fimbriae appears to play a critical role in both the colonization of the human host by Neisseria gonorrhoeae (Gc) and the elaboration of a number of distinct phenotypes, including competence for natural transformation (Seifert et al., 1990; Zhang et al., 1992), twitching motility (Swanson, 1978), autoagglutination (Swanson et al., 1971), and adherence to epithelial tissue (Swanson, 1973; Heckels, 1989; Rudel et al., 1992). Type IV pili, originally defined by their related morphologies and polarized localizations on the cell (Ottow, 1975), have been demonstrated in many other important mucosal pathogens of humans (Hermodson et al., 1978; Strom and Lory, 1986; Giron et al., 1991; Shaw and Taylor, 1990). Studies have shown that this broad classification scheme corresponds well with the presence of a highly conserved $\mathrm{N}$-terminal domain in the structural subunit which accounts for the shared antigenicity and immunogenicity demonstrated by these molecules (Patel et al., 1991). Evidence for the relatedness of these organelles can also be found in the conservation of genes and gene products required for their expression. These components include related prepilin peptidases, soluble proteins with consensus nucleotide-binding motifs and polytopic cytoplasmic membrane proteins but, with the exception of the prepilin peptidases, the functions served by these molecules remains unclear (Lory, 1992; Pugsley, 1993). Homologues corresponding to one or more of these molecules have been implicated in the main terminal branch of the general secretory pathway (GSP (Pugsley, 1993), which is responsible for extracellular localization of a wide variety of toxins and hydrolases by Gram-negative bacteria) and expression of competence for transformation in Bacillus subtilis (Albano et al., 1989). The fundamental 
question then arises as to how these processes might be related to the expression and biogenesis of type IV pili and vice versa.

In the context of Gram-negative species, one obvious connection involves the translocation of large, macromolecular complexes across the outer membrane. This process presumably requires an integral outer membrane component and, based on the conservation of the components detailed above, one might anticipate that structurally related molecules might also be found at this site for each system. Strong candidates for such molecules are homologues of the GspD protein family as exemplified by pIV (engaged in the morphogenesis/export of filamentous phage (Brissette and Russel, 1990)), PulD (required for pullulanase secretion in Klebsiella oxytoca (d'Enfert et al., 1989)) and PilQ (involved in type IV pilus biogenesis in N. gonorrhoeae (Drake and Koomey, 1995)) and Pseudomonas aeruginosa $(\mathrm{Pa})$ (Martin et al., 1993). Furthermore, outer membrane proteins with a high degree of identity to the GspD protein family have also been shown to be active in the type III, contact-depedendent secretion systems (Michiels et al., 1991; Allaoui et al., 1993; Kaniga et al., 1994), competence for transformation in Haemophilus influenzae (Tomb et al., 1991), and secretion of S-layer surface-array subunits in Aeromonas hydrophila (Thomas and Trust, 1995). The GspD homologues all possess Nterminal signal sequences and show high levels of sequence conservation in a C-terminal domain encompassing a 200 -amino-acid span rich in polar residues (Genin and Boucher, 1994).

Insights into the structure/function relationships of these proteins have come most readily from studies of pIV and its role in filamentous phage morphogenesis (Russel, 1991). Using a variety of biochemical techniques, it has been demonstrated that pIV forms a homomultimer composed of 10-12 subunits (Russel and Kazmierczak, 1993; Russel, 1994a; Kazmierczak et al., 1994). In addition, the conserved C-terminal domain of pIV has been shown to mediate its insertion into the outer membrane and nonfunctional pIV missense mutants altered at highly conserved residues in the C-terminus (P375) fail to multimerize despite their membrane localization (Russel, 1994a). Analogous findings relating to $\mathrm{XpsD}$, a GspD homologue involved in hydrolase secretion in Xanthomonas campestris pv. campestris have been reported ( $\mathrm{Hu}$ et al., 1995; Chen et al., 1996). More recent findings in the phage system indicated that the abundance and relative SDS and heat stability of high-molecular-mass (HMM) forms of mutant pIVs correlated with their levels of function (Linderoth et al., 1996). Based on these observations, it was proposed that the pIV multimer forms a channel through which phage particles are assembled and extruded and that the other GspD homologues might form analogous export channels (Russel, 1994b; 1995). Gonococcal PilQ forms a homomultimer composed of 10-12 subunits which is resistant to SDS and heating (Newhall et al., 1980 ) and we have shown that the integrity of the conserved C-terminal residues is necessary for function and detection of the HMM form (Drake and Koomey, 1995). Evidence for SDS- and heat-stable HMM forms of other GspD homologues has also been accumulating (Hardie et al., 1996; Plano and Straley, 1995).

We report here the characterization of the region $1.3 \mathrm{~kb}$ upstream of the $N$. gonorrhoeae pilQ gene and identification of two new genes, pilO and pilP. The product of pilP is a lipoprotein which is essential for pilus biogenesis and HMM PilQ stability. Based on the unique phenotypes found for both $\mathrm{PilP}^{-}$and $\mathrm{PilQ}^{-}$mutants, PilP appears to be required for PilQ function. The product of the pilO gene is necessary for competence for transformation but formal evidence for its role in organelle biogenesis is lacking.

\section{Results}

\section{Nucleotide sequence analysis of the pilQ upstream region}

Gc PilQ ${ }^{-}$mutants are defective in pilus biogenesis (Drake and Koomey, 1995) and it was therefore of interest to examine if other assembly-related genes might be linked to pilQ. As sequence analyses and mutagenesis of the region immediately $3^{\prime}$ to pilQ failed to reveal such genes (Drake and Koomey, 1995), restriction fragments from the cosmid clone pAE8 which hybridized with probes corresponding to the pilQ $5^{\prime}$ end were subcloned into a high-copy-number vector. The nucleotide sequence of the $1.6 \mathrm{~kb}$ EcoRV-Clal fragment in pKSD showed that the clone began at the most $5^{\prime} \mathrm{Clal}$ site in pilQ and extended $1.3 \mathrm{~kb}$ upstream of the gene. Two seemingly significant open reading frames (ORFs) arrayed in the same orientation as pilQ were detected (designated ORFs 5 and 6) and high scoring matches to each were found using the BLASTX program. ORF5, which began at the EcoRV site, was $\approx 33 \%$ identical to the central 80 residues of both the $\mathrm{Pa}$ and Pseudomonas syringae (Ps) PilO proteins (Martin et al., 1995; Roine et al., 1996), while ORF6 was $30 \%$ identical (over 110 residues) to the Pa PilP protein (Martin et al., 1995) and sightly less related to Ps PilP (Roine et al., 1996). Based on the alignment of the most $5^{\prime}$ ORF with $\mathrm{Pa} / \mathrm{Ps} \mathrm{PilO}$, it appeared that part of the ORF corresponding to the PilO $\mathrm{N}$-terminal domain was missing from pKSD. DNA sequencing of a plasmid carrying a $9 \mathrm{~kb} E c o \mathrm{Rl}$ fragment derived from pAE8 showed that a contiguous ORF with N-terminal identity to $\mathrm{Pa} / \mathrm{Ps}$ PilO did in fact span the EcoRV site. Further characterization of this clone showed that sequences starting $0.5 \mathrm{~kb} 5^{\prime}$ of the EcoRV site were different from that present in the Gc genome based on Southern hybridization studies and, 
A

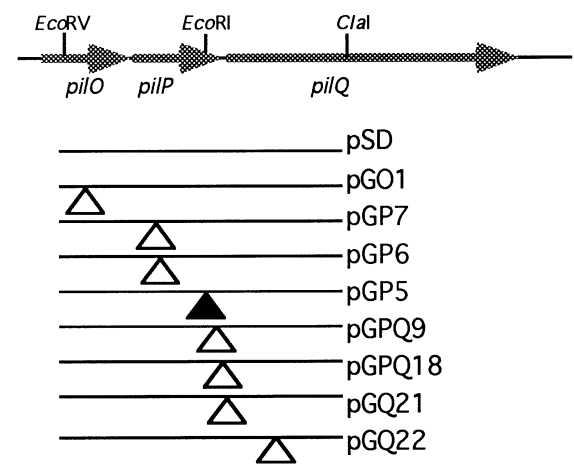

B

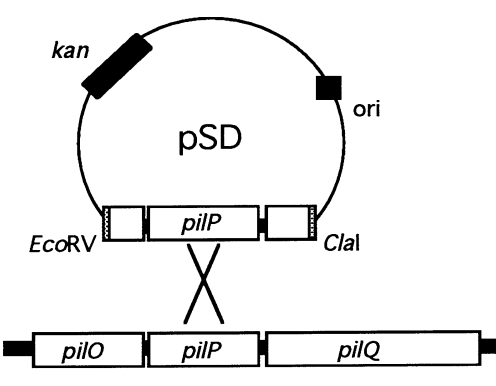

Fig. 1. Genomic organizations and physical maps of the constructs and strains used in this study.

A. Plasmid derivatives of $p S D$ containing transposon insertions (denoted by triangles) and a cat gene cassette insertion (filled triangle at EcoRI site).

B. Depiction of the recombination event leading to integration of $\mathrm{PSD}$ into the genome of N400 to create strain J8. Note that although it is shown as a single cross-over between plasmid and genome, the actual mechanism does not involve a circular form of pSD (see the Discussion).

C. Positions of transposon insertions in strain J8 derivatives which carry a duplication of the pilP gene and flanking region (strain designations are shown below the triangles).

C

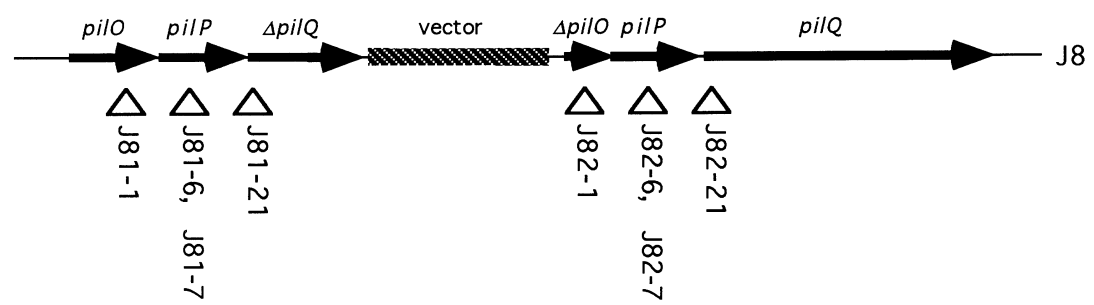

as such, this clone was not used for further characterization of the upstream locus.

ORF5, 645-bp long, potentially encodes a polypeptide of 215 residues with a molecular mass of $23327 \mathrm{Da}$ which, while lacking an apparent signal sequence, would contain one putative membrane-spanning segment near the $\mathrm{N}$-terminus. Other than $\mathrm{Pa} / \mathrm{Ps} \mathrm{PilO}$, no other significant matches were found in the databases. Starting with a initiation codon $18 \mathrm{bp}$ downstream of the termination codon for ORF5 and ending $18 \mathrm{bp} 5^{\prime}$ of the start codon for PilQ, ORF6 consists of $543 \mathrm{bp}$ and encodes a product of 181 residues with a molecular mass of $20110 \mathrm{Da}$. The derived polypeptide of ORF6 contains an N-terminal signal sequence characteristic of lipoproteins with a consensus tetrapeptide cleavage site of LSAC. In addition to the Pa/Ps PilP proteins, the only other potential match to ORF6 was the OspA lipoprotein of Borrelia burgdorferi with which it shares $22 \%$ identity. Overall, the content and organization of the Gc ORF5/ORF6/pilQ locus is strikingly similar to that of the $\mathrm{Pa}$ and Ps pilO, pilP and pilQ loci (Martin et al., 1995; Roine et al., 1996).

\section{Mutagenesis of the pilQ upstream region}

As a first approach, mutagenesis was used to assess if the Gc ORFs did, in fact, correspond to genes and gene products involved in pilus biogenesis. Defined mutations were constructed in Escherichia coli using a transposon shuttle mutagenesis system (Seifert et al., 1986) and these mutations were returned to the genome of Gc strain N400 by transformation. Seven transposon insertion mutants, predicted to disrupt ORF5, ORF6 and the $5^{\prime}$ portion of pilQ, were selected for further study (Fig. 1A). An eighth mutation was constructed by insertion of a promoterless cat gene cassette into the EcoRI site within ORF6. After ensuring the correct introduction of the mutations by Southern hybridization and that no changes in pilE (the pilin subunit gene) had occurred, pilus expression and associated phenotypes were assessed. With one exception, all of the Gc transposon mutants (i) lacked pili detectable by transmission electron microscopy (TEM); (ii) had greatly reduced rates of competence for natural transformation; and (iii) displayed non-autoagglutinating phenotypes. The one exception, strain GPQ18 which carried a transposon insertion 6 bp $5^{\prime}$ of the start codon for PilQ, was piliated (albeit at reduced levels), had wildtype frequencies for transformation and displayed a diminished but readily detectable capacity to autoagglutinate. All of the above mutants released S-pilin (a soluble form of proteolytically degraded PilE lacking the $40 \mathrm{~N}$-terminal residues) into culture supernatants. The strain containing the cat gene-cassette insertion was indistinguishable from the wild-type parent.

\section{PilQ expression in mutant backgrounds}

PilQ has been demonstrated to be essential for pilus expression. To determine whether the non-piliated state 

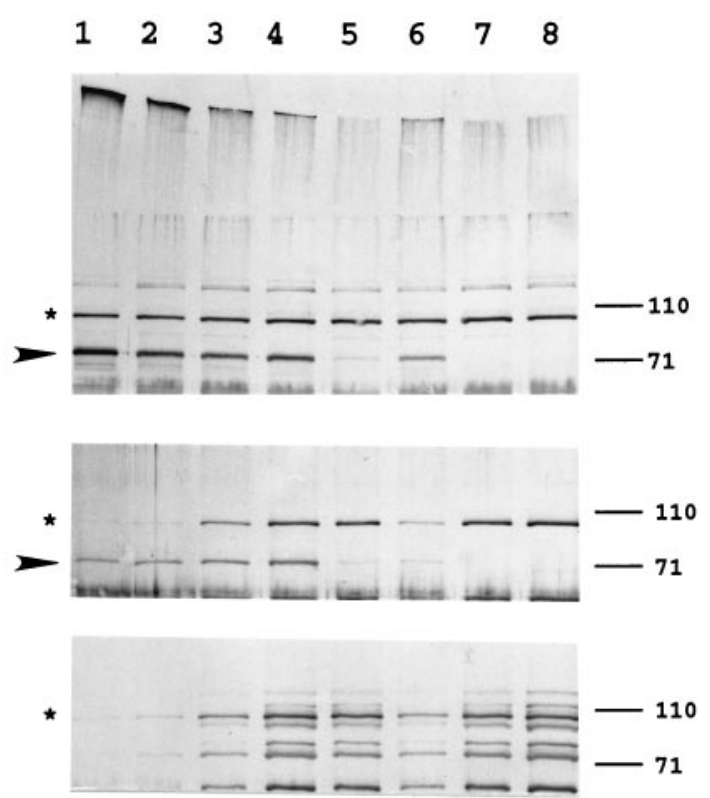

Fig. 2. Detection of PilQ and PilC antigen forms in N400 and derivatives. Lanes: 1, N400; 2, GO1; 3, GP6; 4, GP7; 5, GPQ9; 6, GPQ18; 7, GQ21, 8, GQ22. Top panel, immunoblot of whole-cell lysates using PilQ rabbit antibodies. Reactive material at the top of the panel corresponds to HMM PilQ retained in the well of the stacking gel. Middle panel, immunoblot of soluble proteins in culture supernatants using PilQ rabbit antibodies. Arrows for top and middle panels denote migration of the PilQ monomer $(75 \mathrm{kDa})$. Bottom panel, immunoblot of soluble proteins in culture supernatants using PilC rabbit antibodies. PilC and apparently identically reactive species seen using PilQ antibodies are denoted by asterisks. The relative mobilities of molecular mass standards are shown in $\mathrm{kDa}$.

of the mutants was a consequence of polarity on pilQ expression imposed by the transposon insertions, the levels of PilQ in these backgrounds were examined by immunoblotting (Fig. 2, top panel). GQ21 and GQ22 carry insertions in the pilQ ORF and make no detectable antigen (lanes 7 and 8). GPQ9 and GPQ18, containing insertion mutations $5 \mathrm{bp}$ apart in the $18 \mathrm{bp}$ region between ORF6 and the pilQORF, had greatly reduced levels of the $75 \mathrm{kDa}$ PilQ with the level in GPQ18 being greater than in GPQ9 (lanes 5 and 6). Unless transcription can proceed across the transposons, PilQ expression in these mutants appears to be dependent on promoters within the element and it is worth noting, in this regard, that the transposons in GPQ9 and GPQ18 are in opposite orientations. The mutants with insertions in ORF5 and ORF6 expressed slightly less of the $75 \mathrm{kDa}$ species (lanes 2, 3 and 4) but, given the piliated state of GPQ18 (which expresses far less PilQ; lane 6), their assembly defects cannot be solely due to polarity exerted on pilQ. Thus, a polypeptide corresponding to ORF6 appeared to be required for pilus assembly and, in accord with this and other data, ORF6 was designated pilP and its product PilP. A direct role for a product corresponding to ORF5 cannot be surmised from this data because its transposon insertion mutation might exhibit polarity on pilP. However, based on its relationship to $\mathrm{Pa} / \mathrm{Ps}$ pilO and data detailed below, ORF5 was designated pilO.

\section{Altered forms of PilC and PilQ in mutants}

A significant amount of the Gc PilQ antigen is retained at the buffer/stacking-gel interface following SDS-PAGE (Drake and Koomey, 1995). This unique HMM species of PilQ appears to reflect its propensity to form multimers consisting of 10-12 monomers which can be completely dissociated only after reduction and alkylation (Newhall et al., 1980). In the immunoblot studies examining PilQ expression, we found that the levels of HMM PilQ were reduced in all of the Gc transposon mutants (Fig. 2, top panel). In GPQ9, no antigen in this form could be detected (lane 5), while in GPQ18, HMM PilQ was detected (lane 6) and the relative ratio of the $\mathrm{HMM}$ form to the $75 \mathrm{kDa}$ species was close to that seen in the wild-type parent. However, the ratios of HMM PilQ to monomer were dramatically reduced in both $\mathrm{PilP}^{-}$mutants (lanes 3 and 4). A slight reduction in the level of HMM PilQ relative to monomer was noted in whole-cell lysates of the $\mathrm{PilO}^{-}$insertion mutant (lane 2). Reduced levels of HMM PilQ were not seen in any other class of pilus biogenesis mutants (e.g.

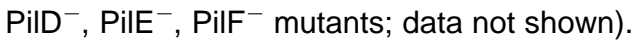

The presence of PilQ in concentrated culture supernatants was examined because mutants expressing truncated (and non-functional) PilQ have been shown to release detectable levels of the antigen (Drake and Koomey, 1995). Culture supernatants of both of the PilPmutants appeared to contain elevated levels of PilQ monomer (Fig. 2, middle panel, lanes 3 and 4). Interestingly, a PilQ antibody cross-reactive protein with a molecular mass of approximately $105 \mathrm{kDa}$ present in whole-cell lysates was seen in culture supernatants of $\mathrm{PilP}^{-}$and $\mathrm{PilQ}^{-}$mutants (denoted by the asterisks in Fig. 2). Additionally, while lower levels of this antigen were detected in the GPQ18 supernatant (lane 6), it was absent from those of the wild type and the $\mathrm{PilO}^{-}$mutant (lanes 1 and 2). Given its molecular mass and altered localization in the mutants, we asked if this molecule might be PilC, an assembly-facilitating protein implicated in pilus-dependent adherence to epithelial cells. A pattern virtually identical to that seen with PilQ antibodies was seen for PilC antibodies with a $105 \mathrm{kDa}$ species being found in culture supernatants of the PilP- and PilQ ${ }^{-}$mutants and reduced amounts of it present in the GPQ18 sample (Fig. 2, bottom panel). This $105 \mathrm{kDa}$ species in supernatants was not seen in any other class of pilus biogenesis mutants (data not shown). As such, PilC appeared to be shed in a soluble form only in $\mathrm{PilQ}^{-}$mutants, mutants expressing reduced amounts of $\mathrm{PilQ}$, and in $\mathrm{PilP}^{-}$mutants. A second 


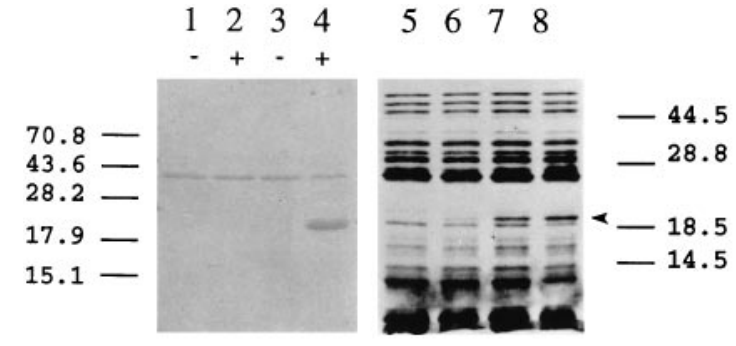

Fig. 3. Autoradiographic detection of PilP by labelling with $\left[{ }^{3} \mathrm{H}\right]-$ palmitic acid. Detection using the T7-based expression system in E. coli BL21(DE3) containing pT7-5 (the vector; lanes 1 and 2) or pT7-P (lanes 3 and 4 ) in the absence $(-)$ and presence $(+)$ of IPTG-induced T7 RNA polymerase. Detection of PilP in Gc strains. Lanes: 5, GP7; 6, GO1; 7, GQ22; 8, N400. The [ $\left.{ }^{3} \mathrm{H}\right]$-palmitic acidlabelled PilP $(\approx 20 \mathrm{kDa})$ is denoted by the arrowhead. The relative mobilities of molecular mass standards are shown in $\mathrm{kDa}$.

protein species with a molecular mass of $58 \mathrm{kDa}$ was also seen with both sera and its presence coincided with that of the $105 \mathrm{kDa}$ antigen. To determine if the two sera were indeed reacting with the same proteins, supernatants from GQ21 were immunoblotted, the filter strip cut to divide the lane in half, and each half reacted with one of the sera. After developing the strips, it was found by re-aligning them that the $105 \mathrm{kDa}$ and $58 \mathrm{kDa}$ proteins observed with each sera were identical within the limitations of the technique (data not shown).

\section{Identification of PilP in E. coli and N. gonorrhoeae}

The T7 expression system (Studier and Moffatt, 1986; Tabor and Richardson, 1985) was used to determine if the product of the Gc pilP gene could be detected in $E$. coli, and if, as predicted by the presence of a consensus signal peptidase II cleavage site, it was a lipoprotein. Cultures of $E$. coli BL21(DE3) with pT7P plasmid (constructed in such a way as to orientate the T7 promoter upstream of the pilP gene) and pT7-5 (the vector) were labelled with $\left[{ }^{3} \mathrm{H}\right]$-palmitic acid. A $20 \mathrm{kDa}$ protein species was detected only in the PT7P sample and its detection was strictly dependent on T7 RNA polymerase induction (Fig. 3, lane 4 ), while a non-specific radiolabelled band of $\approx 38 \mathrm{kDa}$ was observed in all samples. For reasons not yet understood, attempts to visualize a product for pilO using the T7 system and intrinsic amino acid incorporation were unsuccessful (data not shown).

In light of these results, we asked if PilP could be detected using whole-cell labelling of $\mathrm{Gc}$ with $\left[{ }^{3} \mathrm{H}\right]$-palmitic acid. Although many radiolabelled proteins were seen, a species of $20 \mathrm{kDa}$ was found in proteins from the wildtype strain and the $\mathrm{PilQ}^{-}$strain GQ2 but absent from the $\mathrm{PilP}^{-}$transposon insertion mutant (Fig. 3, lanes 5-8). A significant reduction in the level of the same $20 \mathrm{kDa}$ protein was seen in the pilO transposon insertion mutant (lane 6).
The latter finding may be an indication that pilO and pilP are co-transcribed and that the reduction in PilP level in that strain is a consequence of polarity exerted by the upstream transposon insertion.

\section{Analyses of strains carrying tandem duplications of the pilP gene}

When constructing the Gc transposon mutants, we occasionally noted the presence of both piliated and nonpiliated colony types among the primary chloramphenicol-resistant Gc transformants. Examination of the circumstances under which this heterogeneity arose showed that it occurred with $p G O 1$ and $p G Q 22$ plasmid DNAs (Fig. 1A) and only when undigested (i.e. circular) DNA was used. Given that these two plasmids carry transposon insertions close to the ends of the Gc DNA insert in PSD, it seemed that the piliated transformants derived from these constructs might arise by integration of the whole plasmid DNA into the Gc genome (as depicted in Fig. 1B). This was in fact the case because all the chloramphenicolresistant, piliated transformants were resistant to kanamycin (the gene for kanamycin resistance is carried on the vector part of $\mathrm{pSD}$ ) and shown by hybridization analyses to carry a whole copy of the plasmid integrated at the pilP locus (data not shown). By transforming N400 with intact pSD DNA and selecting for kanamycin resistance, we constructed strain $\mathrm{J} 8$ which carries a duplication of the pilP gene separated by a vector junction (Fig. 1C) and is indistinguishable from N400 in piliation and pilusassociated phenotypes.

One consequence of the duplication in $\mathrm{J} 8$ is that both truncated PilQ (from the $5^{\prime}$ pilQ copy) and intact PilQ (from the $3^{\prime}$ complete gene) should be expressed. As seen by immunoblotting, J8 expressed slightly reduced levels of PilQ monomer as well as species of 23 and $20 \mathrm{kDa}$ detectable with PilQ antibodies (Fig. 4, lane 2, asterisks). By transforming J8 with pGQ21 DNA (carrying a $\mathrm{m}-\mathrm{TnCm}$ insertion in the PilQ initiation codon), piliated derivatives, in which the transposon had recombined into the incomplete $5^{\prime}$ pilQ gene copy (J81-21), and nonpliated derivatives, in which the transposon had recombined into the 3' pilQ gene copy (J82-21), were recovered (Fig. 1C). Immunoblotting confirmed that the $23 \mathrm{kDa}$ and $20 \mathrm{kDa}$ antigen forms were products of the defective pilQ allele (Fig. 4, lanes 3 and 4).

Based on the structure of the duplication in strain J8, it became possible to test directly whether the piliation defects associated with the pilO and pilP transposon insertions were a consequence of altered or reduced pilQ expression. J8 derivatives carrying the transposons in the $3^{\prime}$ copies of pilO and pilP (J82-1, J82-6 and J82-7) were indistinguishable from the $\mathrm{J} 8$ parent, formally proving that sufficient levels of PilQ were expressed in the context 
$\begin{array}{llll}1 & 2 & 3 & 4\end{array}$

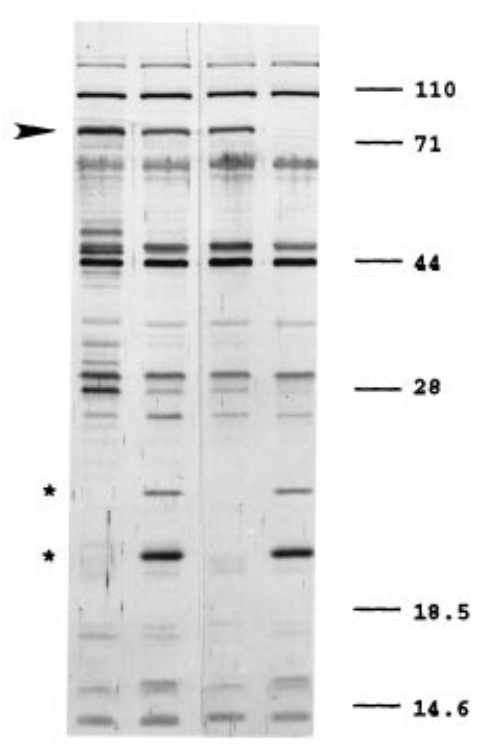

Fig. 4. Immunoblot detection of PilQ antigen forms in whole-cell lysates from strains carrying a duplication of the pilP gene and flanking sequence. Lanes: 1, N400; 2, J8; 3, J81-21; 4, J82-21. The relative mobility of wild-type $\mathrm{PilQ}(75 \mathrm{kDa})$ is denoted by the arrowhead while those of the truncated forms expressed from the defective pilQ allele are indicated by the asterisks. The relative mobilities of molecular mass standards are shown in $\mathrm{kDa}$.

of the insertions (Table 1). Strains J81-6 and J81-7 with the pilP transposon insertions in the upstream gene copy were non-piliated, implying that functional levels of PilP were not expressed from the downstream copy. Strain J81-1, with an insertion in the upstream intact pilO gene copy, was also non-piliated. When competence for transformation was examined, the frequencies for strains J81-7 and $\mathrm{J} 81-6$ were $1 \%$ of that found for piliated derivatives, while the other non-piliated mutants showed approximately 10 -fold lower levels than this $(0.1 \%$ of wild type; Table 1). The intermediate levels of transformability found for J81-7 and J81-6 argue that some functional PilP is expressed from the $3^{\prime}$ gene copy in the J8 backgrounds, while the more absolute defect found for J81-1 proves that PilO plays an essential role in competence and strengthens the concept that it functions in pilus biogenesis.

Radiolabelling of $\mathrm{Gc}$ with $\left[{ }^{3} \mathrm{H}\right]$-palmitic acid was used to assess if PilP was expressed from the $3^{\prime}$ gene copy in the J8 derivatives (Fig. 5). When compared with strain GP7 (which carries an insertion in its single pilP gene copy and makes no product), it is clear that strain J82-7 expresses detectable but significantly reduced levels of the tritiated form of PilP. In addition, the fact that J82-7 expresses less PilP than J82-1 (the $\mathrm{PilO}^{-}$mutant) but has a 10-fold higher level of transformability corroborates the other data, indicating that PilO functions in competence for transformation and, presumably, pilus biogenesis.

\section{Discussion}

This study has led to the identification of two genes which map immediately upstream of the gene that encodes PilQ, an essential pilus biogenesis component and member of the GspD protein family. The deduced polypeptides of the gene ORFs share significant identities with proteins required for pilus expression in $\mathrm{Pa}$ and $\mathrm{Ps}$, the genes for which are arrayed upstream of a gene encoding a GspD homologue (Martin et al., 1995; Roine et al., 1996). The findings extend the previously documented conservation found for pilus-biogenesis components in $\mathrm{Gc}$ and $\mathrm{Pa} / \mathrm{Ps}$. Overall, the gross organization of these particular gene clusters are strikingly similar and recent studies have shown that Gc ORFs corresponding to $\mathrm{Pa} / \mathrm{Ps}$ pilM and pilN can be found immediately $5^{\prime}$ of the pilOgene (S. Dunham and M. Koomey, unpublished). However, subtle differences can be found. For example, in Pseudomonas species, the termination codons of the pilO ORFs overlap the initiation codon for pilP, while in Gc there is a $17 \mathrm{bp}$ intergenic region which posesses a potential ribosomebinding site. Mutant analyses in each system appear to indicate that transcriptional organization of pilO, pilP and pilQ may also be conserved. In $\mathrm{Pa} / \mathrm{Ps}$, pilO and pilP appear to be co-transcribed while pilQ seems to have a functional promoter within the $300 \mathrm{bp}$ region upstream of its initiation codon within pilP (Martin et al., 1995; Roine et al., 1996). Based on previous studies which indicate that the transposons used in this study do

Table 1. Phenotypic characteristics of $N$. gonorrhoeae strains.

\begin{tabular}{lccc}
\hline Strain & Pili $^{\mathrm{a}}$ & Autoagglutination & $\begin{array}{c}\% \text { Natural } \\
\text { competence }\end{array}$ \\
\hline N400 & + & +++ & 100 \\
GO1 & - & - & $<0.1$ \\
GP6 & - & - & $<0.1$ \\
GP7 & - & - & $<0.1$ \\
GP5 & + & +++ & 100 \\
GPQ9 & - & - & $<0.1$ \\
GPQ18 & + & + & 100 \\
GQ21 & - & - & $<0.1$ \\
GQ22 & - & - & $<0.1$ \\
J8 & + & +++ & 100 \\
J81-1 & - & - & $<0.1$ \\
J81-6 & - & - & 1 \\
J81-7 & - & +++ & 100 \\
J81-21 & + & +++ & 100 \\
J81-22 & + & +++ & 100 \\
J82-1 & + & +++ & 100 \\
J82-6 & + & +++ & 100 \\
J82-7 & + & - & $<0.1$ \\
J82-21 & - & - & $<0.1$ \\
J82-22 & - & - & $<0.1$ \\
GF-2 & - & - & \\
\hline
\end{tabular}

a. Determined by transmission electron microscopy.

b. Determined by colony morphology and growth in liquid media.

c. Determined using a DNA transformation assay under RecAinduced conditions.

d. Reduced number of pili noted by TEM.

e. Non-piliated PilF ${ }^{-}$control strain (Freitag et al., 1995). 
$\begin{array}{lllllll}1 & 2 & 3 & 4 & 5 & 6 & 7\end{array}$

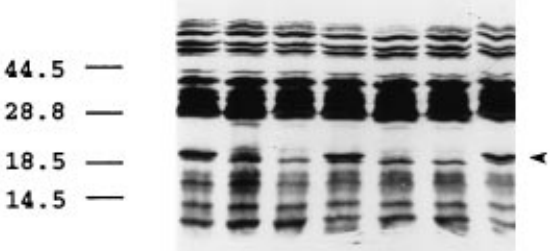

Fig. 5. Autoradiographic detection of $\left[{ }^{3} \mathrm{H}\right]$-palmitic acid-labelled PilP in whole-cell lysates from Gc strains carrying a duplication of the pilP gene and flanking sequence. Lanes: 1, N400; 2, GO1; 3, GP7; 4, J8; 5, J81-1; 6, J81-7; 7, J82- 7. The [ $\left.{ }^{3} \mathrm{H}\right]$-palmitic acid-labelled PilP $(\approx 20 \mathrm{kDa})$ is denoted by the arrowhead. The relative mobilities of molecular mass standards are shown in $\mathrm{kDa}$.

exhibit polarity (Freitag et al., 1995) and the reduced levels of palmitate-labelled PilP in the pilO insertion mutant, the Gc counterparts are probably transcriptionally coupled. This probability is also supported by the low levels of expression of the downstream pilP gene copy in the J8 derivatives. Similarly, the levels of PilQ expressed in the pilP transposon mutants and also from the downstream pilQ gene copy in the J8 derivatives suggest the presence of a distinct pilQ promoter within the $300 \mathrm{bp}$ upstream of its initiation codon. Nonetheless, slightly reduced levels of monomeric $\mathrm{PilQ}$ are found in the pilO and pilP transposon mutants and the $\mathrm{J} 8$ derivatives. This may mean that PilP stabilizes PilQ in a post-translational fashion and/or that correct stoichiometries of each component are required for stabilization. However, evidence for decreased PilQ stability is lacking because an increase in proteolytic degradation products, such as those found in other PilQ mutants (Drake and Koomey, 1995), is not seen in the absence of PilP (data not shown). Alternatively, the reduced PilQ levels in the mutants may reflect a role for pilO/pilP readthrough transcription in activating a pilQ promoter. More studies using gene fusions and RNA analysis are required to further elucidate this situation.

The genomic integration of whole-plasmid DNA was an unanticipated result stemming from this study. This recombination event appeared to result from a single cross-over between the chromosome and a circular plasmid molecule in a Campbell-type integration fashion (as depicted in Fig. $1 \mathrm{~B})$. Intact plasmid DNA is normally rapidly linearized during the uptake process, making it difficult to envision how this process might occur. A similar finding of plasmid integration, made in Neisseria meningitidis, was attributed to a minority of plasmid DNA molecules which remain circular following uptake (van der Ley et al., 1995). However, results using genetically marked constructs indicate that this process does not involve a single cross-over event but utilizes linearized substrates and seems to involve a third DNA molecule in addition to the plasmid and genomic DNA (S. Sandstedt and M. Koomey, unpublished). In addition to using this methodology to create tandem duplications and assess operon structure as described in this study, this technique has been exploited for in situ cloning, creating gene-fusion reporters while retaining an intact copy of the gene of interest, and stable introduction into the genome of foreign (heterologous) genes (M. Koomey, unpublished). Just as these types of manipulations have become part of the standard repertoire for gene manipulation and strain construction in most prokaryotic systems, their introduction to pathogenic Neisseriae species represents a significant advancement, employing a simple technique. This is especially true because the methodology employs a standard cloning vector already in use by many workers.

While these findings and the fact that functional Gc homologues for the $\mathrm{Pa} / \mathrm{Ps}$ pilO and pilP genes exist are interesting, the more pertinent contributions of this work involve the observations of altered localization of PilC in $\mathrm{PilQ}^{-}$and $\mathrm{PilP}^{-}$mutants, aberrant $\mathrm{PilQ}$ behaviour in PilP $^{-}$mutants, together with other evidence that PilP is required for PilQ function. PilC, originally identified in enriched pilus preparations (Jonsson et al., 1991), acts as a facilitator of pilus biogenesis and appears to be responsible for the epithelial cell-binding activity associated with Gc pili (Rudel et al., 1995b). PilC has also been reported to fractionate with the outer membrane and, using antibody-immunogold labelling, an as yet undetermined proportion of PilC can be found associated with the ends of pilus filaments (Rudel et al., 1995b). The aberrant PilC localization found specifically in PilP and PilQ mutants implies that these components interact with one another at the terminal stage of pilus biogenesis. Given that the PilQ serum which detects PilC was raised against the $800 \mathrm{kDa}$ multimer, it is possible that the cross-reaction reflects the presence of PilC in the complex. However, antibodies raised against recombinant meningococcal PilQ expressed in E. coli also display PilC cross-reactivity (T. Tønjum, unpublished). Rudel and colleagues (1995a) have described the presence of a $75 \mathrm{kDa}$ polypeptide in pili purified from $\mathrm{Gc} \mathrm{PilC}^{-}$pseudorevertants expressing high levels of piliation, a peptide which may be PilQ. Although the mutation responsible for alleviating the biogenesis requirement for PilC is undefined, the probability that the $75 \mathrm{kDa}$ polypeptide is PilQ strengthens the notion that these two molecules interact. If PilC and PilQ interactions are essential for pilus biogenesis, it is curious that a PilC-like molecule has yet to be detected in $\mathrm{Pa}$. In fact, although Gc pilin can be expressed as pili in $P$. aeruginosa (Hoyne et al., 1993), it was reported that this species does not possess a PilC homologue (Rudel et al., 1995a). Nonetheless, given the similarities between $\mathrm{Gc} / \mathrm{Pa} / \mathrm{Ps}$ PilP and PilQ and other assembly components, the functional equivalents of PilC vis à vis assembly will probably be found in these Pseudomonas species. 
The evidence in favour of a physiologically significant role for the HMM homomultimers seen for Gc PilQ and its homologues continues to mount. The ability of pIV to form SDS- and heat-resistant HMM species has been shown to correlate with function in phage morphogenesis (Linderoth et al., 1996). SDS- and heat-resistant HMM forms have now also been observed for PuID in Klebsiella oxytoca (Hardie et al., 1996) and YscC in Yersinia pestis (Plano and Straley, 1995), which suggests that this property may be a common feature of this family of molecules. However, the only mutations characterized to date which lead to a reduction or absence of the HMM forms of these homologues map within their respective structural gene. In that sense, Gc PilP appears to be the first molecule identified other than the PulD homologues themselves which significantly influences formation or stability of the HMM species. It is possible that PilP promotes the efficiency or avidity with which PilQ binds to the membrane matrix used for immunodetection. While we cannot rule this out, we would note that we know of no examples in the literature of such a phenomenon. Moreover, recombinant PilQ expressed in the absence of PilP in E. coli binds with high efficiency to PVDF membranes (data not shown).

Recently, Hardie and colleagues (1996) reported that the lipoprotein PulS, essential for pullulanase secretion, was required for outer membrane localization of PuID. They observed no reduction in the level of HMM PuID in a PulS ${ }^{-}$background but noted that membrane-dissociated HMM species consisted solely of stable, proteolytic breakdown products. Based on these findings, PulS was proposed to represent the first member of a new family of periplasmic chaperones required for outer membrane insertion of PilQ-like molecules. It is clear that the absolute pilus defects seen in the Gc PilP- mutants cannot be solely a consequence of the reduced levels of HMM PilQ, given that PilQ mutants expressing lower levels of the HMM species are piliated. The HMM forms seen in PilPmutants appear, therefore, to be defective, perhaps because they are not localized properly, as suggested by the increased levels of soluble PilQ seen in culture supernatants of PilP $^{-}$mutants. Alternatively, or in addition, PilP may actually be an integral component of the macromolecular structure. Attempts to begin to address these possible explanations are now in progress. As noted above, similar explanations could account for the findings in the pullulanase system (Hardie et al., 1996) and, thus, although there is no obvious sequence identity between PulS and Gc PilP, the findings and fundamental basis or observations in the two systems may be in concordance. In most Gram-negative species, lipoprotein localization appears to be dependent on the amino acid residue immediately adjacent to the processing site (Yamaguchi et al., 1988). Similar to the case for PulS (d'Enfert and
Pugsley, 1989), the localization of PilP is probably in the outer membrane, as it does not have an acidic residue at position +2 . Lipoproteins of unknown function (other than an essential role in each cognate system) have been found in association with other type IV pilus biogenesis (Parsot et al., 1991; Stone et al., 1996) and proteinsecretion systems (Michiels et al., 1991; Allaoui et al., 1992; Condemine et al., 1992). Lipoproteins also play essential roles in T-DNA transfer mediated by Agrobacterium tumefaciens (Fernandez et al., 1996) and expression of related pilus conjugation functions (Perumal and Minckley, 1984; Doran et al., 1994; Pohlman et al., 1994). The relevance of the relationships defined in this study and in the pullulanase system between lipoproteins and stabilization of macromolecular complexes required for outer membrane trafficking to these other transport processes remains to be determined.

\section{Experimental procedures}

\section{Bacterial strains, plasmids, and growth conditions}

Bacterial strains and relevant plasmids are detailed in Table 2. Gonococcal strains were propagated on clear solid Gc media at $37^{\circ} \mathrm{C}$ in $5 \% \mathrm{CO}_{2}$ or in media lacking agar that had been preincubated overnight in $5 \% \mathrm{CO}_{2}$ (Koomey et al., 1987). For examination of secreted/released proteins in culture supernatants, chemically defined media (containing free amino acids) was used as previously described (Catlin, 1973). E. coli HB101 (Betheseda Research Laboratories) was used in plasmid-cloning experiments and recombinant strains were grown at $37^{\circ} \mathrm{C}$ in Luria-Bertani (LB) medium supplemented with the appropriate antibiotics. E. coli HB101 was used as the host for subcloning experiments. E. coli BL21(DE3) was used for T7 expression experiments. The DNA sequence for the pilO-pilQ gene cluster is maintained in the EMBL/ GenBank/DDBJ Nucleotide Sequence Data Libraries under Accession Number U40596.

\section{General protocols}

Gonococcal transposon mutants were constructed primarily by shuttle mutagenesis using the minitransposon $\mathrm{m}-\mathrm{TnCm}$ (a derivative of Tn3) as described previously (Seifert et al., 1986). The precise sites of insertions were determined by DNA sequencing with primer Tn3L (5'-CTCATGACCAAAATCCC- $3^{\prime}$ ) which is complementary to sequences at the left end of the transposon. These and other mutations were introduced into N400 by transformation using plasmid DNAs as detailed previously (Tønjum et al., 1995) and selection for chloramphenicol resistance $\left(10 \mu \mathrm{g} \mathrm{ml}^{-1}\right)$. Gc strain $\mathrm{J} 8$ was generated by transformation of N400 with pSD DNA and selection for resistance to kanamycin $\left(50 \mu \mathrm{g} \mathrm{ml}^{-1}\right)$ and the correct introduction of the plasmid into the genome was verified by Southern hybridization. The $\mathrm{N} 400$ strain harbours the recA6 allele in which the recA gene is under the control of an inducible tac-lac promoter (Freitag et al., 1995). For transformation, RecA expression and recombination functions were 
Table 2. Bacterial strains and plasmids used in this study.

\begin{tabular}{|c|c|c|}
\hline Strain / Plasmid & Relevant characteristics & $\begin{array}{l}\text { Source/ } \\
\text { Reference }\end{array}$ \\
\hline \multicolumn{3}{|l|}{ Strain } \\
\hline \multicolumn{3}{|l|}{$\overline{N . \text { gonorrhoeae }}$} \\
\hline N400 & $\begin{array}{l}\text { Derived from VD } 300^{\mathrm{a}} \text {. Contains } \\
\text { the recA6 allele (IPTG inducible) }\end{array}$ & $\begin{array}{l}\text { Tønjum et al. } \\
\text { (1995) }\end{array}$ \\
\hline GO1 & pilO::m-TnCm at position $365^{\mathrm{b}}$ & This work \\
\hline GP6 & pilP::m-TnCm at position $962^{\mathrm{b}}$ & This work \\
\hline GP7 & pilP::m-TnCm at position $931^{\mathrm{b}}$ & This work \\
\hline GP5 & $\begin{array}{l}\text { pilP::cat (using EcoRI site at } \\
\text { position } 1178^{\mathrm{b}} \text { ) }\end{array}$ & This work \\
\hline GPQ9 & $\mathrm{m}-\mathrm{TnCm}$ at position $1235^{\mathrm{b}}$ & This work \\
\hline GPQ18 & $\mathrm{m}-\mathrm{Tn} C m$ at position $1240^{\mathrm{b}}$ & This work \\
\hline GQ21 & pilQ::m-TnCm at position $1250^{\mathrm{b}}$ & This work \\
\hline GQ22 & pilQ::m-Tn $C m$ at position $1420^{b}$ & This work \\
\hline GQ1 & pilQ::m-TnErm at position $2516^{\mathrm{b}}$ & $\begin{array}{l}\text { Drake and } \\
\text { Koomey } \\
(1995)\end{array}$ \\
\hline J8 & N400 containing integrated pSD & This work \\
\hline $\mathrm{J} 81-1$ & $\begin{array}{l}\mathrm{J} 8 \text { with pilO::m-TnCm at position } \\
365^{\mathrm{c}}\end{array}$ & This work \\
\hline J81-6 & $\begin{array}{l}\text { J8 with pilP::m-TnCm at position } \\
962^{\mathrm{C}}\end{array}$ & This work \\
\hline J81-7 & $\begin{array}{l}\mathrm{J} 8 \text { with pilP::m-TnCm at position } \\
931^{\mathrm{c}}\end{array}$ & This work \\
\hline J81-21 & $\begin{array}{l}\text { J8 with pilQ::m-TnCm at position } \\
1250^{\mathrm{C}}\end{array}$ & This work \\
\hline J82-1 & $\begin{array}{l}\text { J8 with pilO::m-TnCm at position } \\
365^{\mathrm{c}}\end{array}$ & This work \\
\hline J82-6 & $\begin{array}{l}\text { J8 with pilP::m-TnCm at position } \\
962^{\mathrm{C}}\end{array}$ & This work \\
\hline J82-7 & $\begin{array}{l}\mathrm{J} 8 \text { with pilP::m-TnCm at position } \\
931^{\mathrm{C}}\end{array}$ & This work \\
\hline J82-21 & $\begin{array}{l}\text { J8 with pilQ::m-TnCm at position } \\
1250^{\mathrm{C}}\end{array}$ & This work \\
\hline \multicolumn{3}{|l|}{ E. coli } \\
\hline BL21 (DE3) & $\begin{array}{l}\mathrm{F}^{-} \text {omp } T, \mathrm{r}_{\mathrm{B}}^{-} \mathrm{m}_{\mathrm{B}}^{-} \text {with prophage } \\
\lambda \text { carrying the } \mathrm{T} 7 \mathrm{RNA} \\
\text { polymerase gene }\end{array}$ & $\begin{array}{l}\text { Studier et al. } \\
\quad(1990)\end{array}$ \\
\hline HB101 & $\begin{array}{l}\mathrm{F}^{-}, \text {mcrB mrr hsdS20 }\left(\mathrm{r}_{\mathrm{B}}^{-} \mathrm{m}_{\mathrm{B}}^{-}\right) \\
\text {recA13 supE44 ara14 galK2 } \\
\text { lacY1 proA2 rpsL20 }\left(\mathrm{Sm}^{\mathrm{R}}\right) \\
\text { xyl15 } \mathrm{I}^{-} \text {leu } \mathrm{mtl1}\end{array}$ & Gibco BRL \\
\hline \multicolumn{3}{|l|}{$\underline{\text { Plasmid }}$} \\
\hline$\overline{\mathrm{pAE} 8}$ & Cosmid clone & $\begin{array}{l}\text { Drake and } \\
\text { Koomey } \\
(1995)\end{array}$ \\
\hline $\begin{array}{l}\text { pBluescript-II } \\
\text { SK+ }\end{array}$ & Cloning vector & Stratagene \\
\hline $\mathrm{pKSD}$ & $\begin{array}{l}\text { EcoRV-Clal } 1.6 \mathrm{~kb} \text { fragment in } \\
\text { pBluescript-II SK+ }\end{array}$ & This work \\
\hline pSD & $\begin{array}{l}\text { EcoRV-Clal } 1.6 \mathrm{~kb} \text { fragment in } \\
\text { pHSS6 }\end{array}$ & This work \\
\hline pGP5 & $\begin{array}{l}\text { EcoRV-Cla+l } 1.6 \mathrm{~kb} \text { containing } \\
\text { cat cassette (derived from } \\
\text { pCM7) insertion at the EcoRI } \\
\text { site }\end{array}$ & This work \\
\hline pT7-5 & $\begin{array}{l}\text { T7 RNA polymerase promoter } \\
\text { f10, Ap }\end{array}$ & $\begin{array}{l}\text { Tabor and } \\
\text { Richardson } \\
(1985)\end{array}$ \\
\hline pT7P & $\begin{array}{l}\text { Xhol (EcoRV)-Clal fragment } \\
\text { (derived from pKSD) in pT7-5 }\end{array}$ & This work \\
\hline
\end{tabular}

a. VD300 is a MS11 derivative (Koomey et al., 1987)

b. Derived by transformation using PSD derivative plasmid DNAs (Figs 1 and 2). Nucleotide positions correspond to those in EMBL/ Genbank/DDBJ Nucleotide Sequence Data Libraries under Accession Number U40596.

c. Strains contain insertions $5^{\prime}(\mathrm{J} 81-)$ or $3^{\prime}$ (J82-) of the integrated vector. Locations of transposon insertions are shown in Fig. 2. transiently induced in this background by IPTG addition to a final concentration of $200 \mu \mathrm{M}$ at the same time at which transforming DNA was added.

The complete sequences of cloned DNAs were determined using the dideoxy chain-termination method (Sanger et al., 1977) with Sequenase (USB) in conjunction with standard vector-based and customized oligonucleotide primers. The pilE (pilin subunit-encoding) gene nucleotide sequence of each mutant was determined by thermocycle sequencing of polymerase chain reaction (PCR) products using CircumVent polymerase (New England Biolabs) and customized oligonucleotide primers. DNA and protein sequence data was compiled and analysed by computer using both the MacVector 3.5 (International Biotechnologies Inc.) and the University of Wisconsin Genetics Computer Group (UWGCG) software packages (Devereux et al., 1984). Homology searches using the sequenced DNA and translated ORFs were performed by accessing the BLAST network server at the National Centre for Biotechnology Information (Gish and States, 1993).

\section{Gonococcal transformation competence assay}

Competence for natural transformation of gonococcal strains was assessed as described using chromosomal DNA from spontaneous rifampicin- and naladixic acid-resistant mutants of VD300 (Zhang et al., 1992). RecA expression and recombination functions were transiently induced by IPTG addition to a final concentration of $200 \mu \mathrm{M}$ at the same time at which transforming DNA was added (see above).

\section{Transmission electron microscopy}

Electron microscopy samples were prepared by placing carbon-coated Formvar $3.05 \mathrm{~mm}$ grids (Tousimas Research Corp.) onto individual bacterial colonies propagated for $16 \mathrm{~h}$. Grids were air dried for $10 \mathrm{~min}$, floated on a drop of $0.25 \%$ sodium phosphotungstic acid (NaPT), $\mathrm{pH} 7.0$, for $30 \mathrm{~s}$ and air dried again. Grids were examined using a Philips CM-10 Transmission Electron Microscope.

\section{SDS-PAGE and immunoblotting}

The presence of PilE, PilQ and PilC in whole-cell lysates and concentrated culture supernatants was detected by immunoblotting using rabbit polyclonal antibodies as described previously (Drake and Koomey, 1995) and rabbit polyclonal PilC-specific antibodies (a gift of T. F. Meyer). Antigen detection was performed by use of alkaline phosphatase-coupled goat anti-rabbit immunoglobulin antibodies (Tago Inc.) and a colourimetric alkaline phosphate substrate. Conditions for sample preparation, SDS-PAGE, membrane transfer and antigen detection have been described previously (Blake et al., 1984). Proteins were transferred to Immobilon-PSQ PVDF membranes (Millipore Corp.). Pre-stained molecular weight protein standards (Diversified Biotech and Gibco BRL) were used).

\section{$\left[{ }^{3} \mathrm{H}\right]$-palmitic acid labelling of lipoproteins}

$\left[{ }^{3} \mathrm{H}\right]$-palmitic acid (specific activity $50 \mathrm{Ci} \mathrm{mM}^{-1}$; Amersham) was concentrated by evaporation of solvent and added to 
cultures to achieve a concentration of $20 \mu \mathrm{Ci} \mathrm{ml}^{-1}$. The PilP protein, expressed in E. coli by using coupled T7 RNA polymerase-promoter gene fusions in strain BL21 (DE3; Table 2 ), was labelled under standard conditions used for amino acid incorporation with the exception that LB medium (rather than defined minimal media) was used. Gonococcal liquid cultures were started using suspensions of fresh (16-h old) plate-grown cells diluted to give an initial concentration of $5 \times 10^{6}$ colony-forming units (cfu) $\mathrm{ml}^{-1}$ and grown for $5 \mathrm{~h}$ at $37^{\circ} \mathrm{C}$ in the presence of $\left[{ }^{3} \mathrm{H}\right]$-palmitic acid. Proteins of wholecell lysates were separated by SDS-PAGE, transferred to Immobilon-PSQ PVDF membranes (Millipore Corp.) and treated with Enhance (Dupont-NEN) for fluorography, as recommended by the manufacturer. Autoradiography was performed over 2-21d using Kodak X-ray films.

\section{Acknowledgements}

We are grateful to C. Wilde III for the gonococcal PilQ-specific polyclonal antibodies, T. F. Meyer for the anti-PilC-specific polyclonal antibodies and M. Sandkqvist, D. Higgins and M. Russel for comments on the manuscript. This work was supported by Public Health Service Grant Al27837 (M.K.), National Institutes of Health (NIH) Clinical Research Center (CRC) grant M01 RR 00042, F32 Al09306 (S.D.), and NIH training grant T32 8I 07360 (S.D.). M.K. was supported, in part, during preparation of this manuscript by a guest researcher stipend from the Norwegian Research Council (Norges forskningråd), project number 109399/300.

\section{References}

Albano, M., Breitling, R., and Dubnau, D.A. (1989) Nucleotide sequence and genetic organization of the Bacillus subtilis comG operon. J Bacteriol 171: 5386-5404.

Allaoui, A., Sansonetti, P.J., and Parsot, C. (1992) MxiJ, a lipoprotein involved in secretion of Shigella Ipa invasins, is homologous to YscJ, a secretion factor of the Yersinia Yop proteins. J Bacteriol 174: 7661-7669.

Allaoui, A., Sansonetti, P.J., and Parsot, C. (1993) MxiD, an outer membrane protein necessary for the secretion of the Shigella flexneri lpa invasins. Mol Micriobiol 7: 59-68.

Blake, M.S., Johnston, K.H., Russell, J.G., and Gotschlich, E.C. (1984) A rapid, sensitive method for detection of alkaline phosphatase-conjugated anti-antibody on Western blots. Anal Biochem 136: 175-179.

Brissette, J.L., and Russel, M. (1990) Secretion and membrane integration of a filamentous phage-encoded morphogenetic protein. J Mol Biol 211: 565-580.

Catlin, B.W. (1973) Nutritional profiles of Neisseria gonorrhoeae, Neisseria meningitidis, and Neisseria lactamica in chemically defined media and the use of growth requirements for gonococcal typing. J Infect Dis 128: 178-194.

Chen, L., Chen, D., Miaw, J., and Hu, N. (1996) XpsD, an outer membrane protein required for protein secretion by Xanthomonas campestris pv. campestris, forms a multimer. J Biol Chem 271: 2703-2708.

Condemine, G., Dorel, C., Hugouvieux-Cotte-Pattat, N., and Robert-Baudouy, J. (1992) Some of the out genes involved in the secretion of pectate lyases in Erwinia chrysanthemi are regulated by $k d g R$. Mol Microbiol 6: 3199-3211.
Devereux, J., Haeberli, P., and Smithies, O. (1984) A comprehensive set of sequence analysis programs for the VAX. Nucleic Acids Res 12: 387-395.

Doran, T.J., Loh, S.M., Firth, N., and Skurray, R.A. (1994) Molecular analysis of the $\mathrm{F}$ plasmid traVR region: traV encodes a lipoprotein. J Bacteriol 176: 4182-4186.

Drake, S.L., and Koomey, M. (1995) The product of the pilQ gene is essential for the biogenesis of type IV pili in Neisseria gonorrhoeae. Mol Microbiol 18: 975-986.

d'Enfert, C., and Pugsley, A.P. (1989) Klebsiella pneumoniae pulS gene encodes an outer membrane lipoprotein required for pullulanase secretion. J Bacteriol 171: 36733679.

d'Enfert, C., Reyss, I., Wandersman, C., and Pugsley, A.P. (1989) Protein secretion by Gram-negative bacteria. Characterization of two membrane proteins required for pullulanase secretion by Escherichia coli K-12. J Biol Chem 264: 17462-17468.

Fernandez, D., Spudich, G.M., Zhou, X.-R., and Christie, P.J. (1996) The Agrobacterium tumefaciens VirB7 lipoprotein is required for stabilization of VirB proteins during assembly of the T-DNA complex transport apparatus. J Bacteriol 178: 3168-3176.

Freitag, N., Seifert, H.S., and Koomey, M. (1995) Characterization of the pilF-pilD pilus assembly locus of Neisseria gonorrhoeae. Mol Microbiol 16: 575-586.

Genin, S., and Boucher, C.A. (1994) A superfamily of proteins involved in different secretion pathways in Gramnegative bacteria: modular structure and specificity of the N-terminal domain. Mol Gen Genet 243: 112-118.

Giron, J.A., Ho, A.S.Y., and Schoolnik, G.K. (1991) An inducible bundle-forming pilus of enteropathogenic Escherichia coli. Science 254: 710-713.

Gish, W., and States, D.J. (1993) Identification of protein coding regions by database similarity search. Nat Genet 3: 266-272.

Hardie, K.R., Lory, S., and Pugsley, A.P. (1996) Insertion of an outer membrane protein in Escherichia coli requires a chaperone-like protein. EMBO J 15: 978-988.

Heckels, J.E. (1989) Structure and function of pili of pathogenic Neisseria species. Clin Microbiol Rev 2: S66-S73.

Hermodson, M.A., Chen, K.C., and Buchanan, T.M. (1978) Neisseria pili proteins: amino-terminal amino acid sequences and identification of an unusual amino acid. Biochemistry 17: 442-445.

Hoyne, P.A., Hass, R., Meyer, T.F., Davies, J.K., and Elleman, T.C. (1993) Production of Neisseria gonorrhoeae pili (fimbriae) in Pseudomonas aeruginosa. J Bacteriol 174: $7321-7327$.

Hu, N., Hung, M., Liao, C., and Lin, M. (1995) Subcellular location of $\mathrm{XpsD}$, a protein required for extracellular protein secretion by Xanthomonas campestris pv. campestris. Microbiology 141: 1395-1406.

Jonsson, A.-B., Nyberg, G., and Normark, S. (1991) Phase variation of gonococcal pili by frameshift mutation in pilC, a novel gene for pilus assembly. EMBO J 10: 477488.

Kaniga, K., Bossio, J.C., and Galan, J.E. (1994) Salmonella typhimurium invasion genes invF and invG encode homologues to the PulD and AraC family of proteins. Mol Microbiol 13: 555-568. 
Kazmierczak, B.I., Mielke, D.L., and Russel, M. (1994) pIV, a filamentous phage protein that mediates phage export across the bacterial cell envelope, forms a multimer. $J$ Mol Biol 238: 187-198.

Koomey, M., Gotschlich, E.C., Robbins, K., Bergstrom, S., and Swanson, J. (1987) Effects of recA mutations on pilus antigenic variation and phase transitions in Neisseria gonorrhoeae. Genetics 117: 391-398.

van der Ley, P., van der Biezen, J., and Poolman, J.T. (1995) Construction of Neisseria meningitidis strains carrying multiple chromosomal copies of the porA gene for use in the production of a multivalent outer membrane vesicle vaccine. Vaccine 13: 401-407.

Linderoth, N.A., Model, P., and Russel, M. (1996) Essential role of a sodium dodecyl sulfate-resistant protein IV multimer in assembly export of filamentous phage. $J$ Bacteriol 178: 1962-1970.

Lory, S. (1992) Determinants of extracellular protein secretion in Gram-negative bacteria. J Bacteriol 174: 34233428.

Martin, P.R., Hobbs, M., Free, P.D., Jeske, Y., and Mattick, J.S. (1993) Characterization of pilQ, a new gene required for the biogenesis of type 4 fimbriae in Pseudomonas aeruginosa. Mol Microbiol 9: 857-868.

Martin, P.R., Watson, A.A., McCaul, T.F., and Mattick, J.S. (1995) Characterization of a five-gene cluster required for the biogenesis of type 4 fimbriae in Pseudomonas aeruginosa. Mol Microbiol 16: 497-508.

Michiels, T., Vanooteghem, J.C., Lambert de Rouvroit, C., China, B., Gustin, A., Boudry, P., and Cornelis, G.R. (1991) Analysis of virC, an operon involved in the secretion of Yops proteins by Yersinia enterocolitica. J Bacteriol 173: 4994-5009.

Newhall, W.J., Wilde, C.E., Sawyer, W.D., and Haak, R.A. (1980) High-molecular-weight antigenic protein complex in the outer membrane of Neisseria gonorrhoeae. Infect Immun 27: 475-482.

Ottow, J.C.G. (1975) Ecology, physiology, and genetics of fimbriae and pili. Annu Rev Microbiol 29: 79-108.

Parsot, C., Taxman, E., and Mekalanos, J.J. (1991) ToxR regulates the production of lipoproteins and the expression of serum resistance in Vibrio cholerae. Proc Natl Acad Sci USA 88: 1641-1645.

Patel, P., Marrs, C.F., Mattick, J.S., Ruehl, W.W., Taylor, R.K., and Koomey, M. (1991) Shared antigenicity and immunogenicity of type IV pilins expressed by Pseudomonas aeruginosa, Moraxella bovis, Neisseria gonorrhoeae, Dichelobacter nodosus and Vibrio cholerae. Infect Immun 59: 4674-4676.

Perumal, N.B., and Minckley, E.G.J. (1984) The product of the $\mathrm{F}$ sex factor traT surface exclusion gene is a lipoprotein. J Biol Chem 259: 5357-5360.

Plano, G.V., and Straley, S.C. (1995) Mutations in yscC, $y s c D$, and $y s c G$ prevent high-level expression and secretion of $\mathrm{V}$ antigen and Yops in Yersinia pestis. J Bacteriol 177: 3843-3854.

Pohlman, R.F., Genetti, H.D., and Winans, S.C. (1994) Common ancestry between IncN conjugal transfer genes and macromolecular export systems of plant and animal pathogens. Mol Microbiol 14: 655-668.

Pugsley, A.P. (1993) The complete general secretory pathway in Gram-negative bacteria. Microbiol Rev 57: 50-108.

Roine, E., Nunn, D.N., Paulin, L., and Romantschuk, M. (1996) Characterization of genes required for pilus expression in Pseudomonas syringae pathovar phaseolicola. $J$ Bacteriol 178: 410-417.

Rudel, T., van Putten, J.P.M., Gibbs, C.P., Hass, R., and Meyer, T.F. (1992) Interaction of two variable proteins (PilE and PilC) required for pilus-mediated adherence of Neisseria gonorrhoeae to human epithelial cells. Mol Microbiol 6: 3439-3450.

Rudel, T., Boxberger, H.-J., and Meyer, T.F. (1995a) Pilus biogenesis and epithelial cell adherence of Neisseria gonorrhoeae pilC double knock-out mutants. Mol Microbiol 17: 1057-1071.

Rudel, T., Scheuerpflug, I., and Meyer, T.F. (1995b) Neisseria PilC protein identified as type-4 pilus tip-located adhesin. Nature 373: 357-359.

Russel, M. (1991) Filamentous phage assembly. Mol Microbiol 5: 1607-1613.

Russel, M. (1994a) Mutants at conserved positions in gene IV, a gene required for assembly and secretion of filamentous phages. Mol Microbiol 14: 357-369.

Russel, M. (1994b) Phage assembly: a paradigm for bacterial virulence factor export. Science 265: 612-614.

Russel, M. (1995) Moving through the membrane with filamentous phages. Trends Microbiol 3: 223-228.

Russel, M., and Kazmierczak, B. (1993) Analysis of the structure and subcellular location of filamentous phage pIV. $J$ Bacteriol 175: 3998-4007.

Sanger, F., Nicklen, S., and Coulson, A.R. (1977) DNA sequencing with chain-terminating inhibitors. Proc Natl Acad Sci USA 74: 5463-5467.

Seifert, H.S., Chen, E.Y., So, M., and Heffron, F. (1986) Shuttle mutagenesis: a method of transposon mutagenesis for Saccharomyces cerevisiae. Proc Natl Acad Sci USA 83: 735-739.

Seifert, H.S., Ajioka, R.S., Paruchuri, D., Heffron, F., and So, M. (1990) Shuttle mutagenesis of Neisseria gonorrhoeae: pilin null mutations lower DNA transformation competence. $J$ Bacteriol 172: 40-46.

Shaw, C.E., and Taylor, R.K. (1990) Vibrio cholerae 0395 tcpA pilin gene sequence and comparison of the predicted protein structural features to those of type IV pilins. Infect Immun 58: 3042-3049.

Stone, K.D., Zhange, H., Carlson, L.K., and Donnenberg, M.S. (1996) A cluster of fourteen genes from enteropathogenic Escherichia coli is sufficient for the biogenesis of a type IV pilus. Mol Microbiol 20: 325-337.

Strom, M.S., and Lory, S. (1986) Cloning and expression of the pilin gene of Pseudomonas aeruginosa PAK in Escherichia coli. J Bacteriol 165: 367-372.

Studier, F.W., and Moffatt, B.A. (1986) Use of bacteriophages T7 RNA polymerase to direct selective high-level expression of cloned genes. J Mol Biol 189: 113-130.

Swanson, J. (1973) Studies on gonococcus infection. IV. Pili: their role in attachment of gonococci to tissue culture cells. J Exp Med 137: 571-589.

Swanson, J. (1978) Studies on gonococcus infection XII. Colony color and opacity variants of gonococci. Infect Immun 19: 320-331. 
Swanson, J., Kraus, S.J., and Gotschlich, E.C. (1971) Studies on gonococcus infection. I. Pili and zones of adhesion: their relation to gonococcal growth patterns. $J$ Exp Med 134: 886-906.

Tabor, S., and Richardson, C.C. (1985) A bacteriophage T7 RNA polymerase/promoter specific system for controlled exclusive expression of specific genes. Proc Natl Acad Sci USA 82: 1074-1078.

Thomas, S.R., and Trust, R.J. (1995) A specific PulD homolog is required for the secretion of paracrystalline surface array subunits in Aeromonas hydrophilia. J Bacteriol 177: 3932-3939.

Tomb, J., El-Hajj, H., and Smith, H.O. (1991) Nucleotide sequence of a cluster of genes involved in the transformation of Haemophilus influenzae Rd. Gene 104: 1-10.

Tønjum, T., Freitag, N.E., Normark, E., and Koomey, M. (1995) Identification and characterization of pilG, a highly conserved pilus assembly gene in pathogenic Neisseria. Mol Microbiol 16: 451-464.

Yamaguchi, K., Yu, F., and Inouye, M. (1988) A single amino acid determinant of membrane localization of lipoproteins in E. coli. Cell 53: 423-432.

Zhang, Q.Y., DeRyckere, D., Lauer, P., and Koomey, M. (1992) Gene conversion in Neisseria gonorrhoeae: evidence for its role in pilus antigenic variation. Proc Natl Acad Sci USA 89: 5366-5370. 